\title{
Why Free Will Is Logically Not Possible within Naturalism
}

\author{
Michael Prost \\ Fern Universität in Hagen
}

\begin{abstract}
One of the most intriguing problems of philosophy and of mankind is the question whether humans have a free will. This question is heavily disputed between natural scientists and especially neuroscientists, who deny free will, and philosophers and other groups, who insist on free will. It is perplexing that both sides base their premise on the same precondition, namely naturalism. We will prove that naturalism automatically leads to physicalism, to materialism, and to reductionism. We will also prove here that it is logically not possible to have a free will if naturalism is true. Free will definitely requires an additional substance, a non-material soul, which cannot be part of our universe. This must not be in contradiction to our current knowledge of natural sciences.
\end{abstract}

Keywords: free will, naturalism, physical realization of will decisions, determinism/indeterminism, compatibilism, libertarianism

\section{The Status of the Discussion about Free Will}

The question whether humans have a free will is one of the most intriguing questions of mankind. The human value system is based on the assumption that humans have a free will. Free will is the foundation of human dignity. Even Immanuel Kant already made the very true statement that humans can only be moral creatures if they have a free will (Kant 2008). Consequently, it is not surprising that the big majority of philosophers defend this position. This is not difficult to do because everybody has the feeling of having free will. However, the position became more difficult to defend once physics started to claim that the development of the universe including humans is strictly deterministic. A strict determination of the universe including humans could make free will impossible. This is the reason why the group of modern philosophers who defend free will is split in two groups. One group believes that the claim of determinism is correct. Nevertheless they argue that free will is compatible with determinism. This view is called compatibilism. Another group denies determinism. They argue that free will can never be compatible with determinism. Because they insist on a free will, they refute determinism. This can be reasonable even from a physical perspective because the physical view of quantum theory seems to allow some indeterminacy. This second view is called libertarianism. Prominent propagators of compatibilism are Harry Frankfurt (1988), Alfred Mele (2006), and Daniel Dennett (2002; 2004). Propagators of libertarianism are Carl Ginet (1990; 2002), Robert Kane (2002; 2005), Randolph Clarke (2002; 2005), and Timothy O'Connor (2002/1; 2002/2). An additional perspective came into the discussion by way of neuroscientists who have found out that decision making is only partly conscious, but also partly unconscious (Libet 2005; Roth 2013). Consequently the neuroscientists argue that a mental process such as decision-making that might be dominated by unconsciousness cannot be considered as free. This claim has

Michael Prost, Dr., Institute of Philosophy, Fern Universität in Hagen, Germany; main research field: Philosophy of Mind, Philosophy of Physics, and Neuroscience of Consciousness. 
intensified the controversy. It has to be mentioned here that some philosophers like Galen Strawson (2002; 2010) also support the position of neuroscience and deny freedom of will.

We will show here that the entire discussion between philosophers and neuroscientists is futile. It doesn't play any role for the question about free will what the proportion of unconsciousness in decision-making is. We will show that even if decisions were made completely consciously, they could never be free. The question about the truth of determinism or indeterminism is also irrelevant. In every case will decisions can never be free. The only possibility for the existence of free will would be the existence of an immaterial entity like a soul. We will first explain what the view of naturalism is. Then we will define what free will means and why neither determinism nor indeterminism makes free will possible. Finally, we will outline that one could imagine an extended version of naturalism which would include the existence of a non-material soul. This assumption need not be in contradiction to naturalism and could provide free will. We can call naturalism without soul "pure naturalism" and naturalism with soul "religious naturalism."

\section{Naturalism}

First we must clarify what the philosophical view of naturalism is. Naturalism means that everything that exists was created in a natural way and can be explained by natural sciences. The intention of naturalism is to exclude every non-natural entity from all explanations. This view is especially directed against any religious views. A naturalist would claim that the universe was created in the big bang which was not caused by God. A naturalist would claim that the universe evolved from the big bang following general laws of nature. A naturalist would claim that part of this evolution was the development of our sun, earth, life on earth, and eventually humans. A naturalist would claim that God did not play a part in the evolution of humans and did not give humans a non-material entity such as the soul. Actually, it should be mentioned that naturalism is a minority view. Probably $80 \%$ of the world's population believes in the existence of a non-material soul. It has to be emphasized that the account of the Abrahamic religions is not in complete contradiction to naturalism and is difficult to disprove by science. More so, the account of the Abrahamic religions incorporates the biggest parts of naturalism. The only difference to pure naturalism is that it is assumed that God indeed caused the big bang. Actually, this would solve a huge problem intrinsic to pure naturalism. It is known that there exist 18 natural constants, which are extremely fine-tuned. This means that they show just the necessary values that were needed so that our universe could develop to the universe we experience today. If just one of these 18 natural constants were a little bit off our universe could not exist in its current form and humans would not exist either. Naturalism concedes that this fine-tuning could not have happened by chance; the probability therefore is too small. The only possible explanation physics has so far is the so-called inflation hypothesis. The inflation hypothesis assumes that our universe is part of a multiverse, in which permanently an almost infinite number of new universes is being created. The huge number then makes it possible that one universe, namely ours, has the fine-tuning by accident. Right now there is no reason to claim that the inflation hypothesis is more probable than the claim of the Abrahamic religions that God created the universe via the big bang and that God adjusted all the parameters in a way that led to our universe including humans. In the author's opinion, it is possible to be a naturalist but leave the question open whether the big bang was caused by God or has unknown physical causes. One could call the first view "religious naturalism," the second one "pure naturalism." In both cases, naturalism would claim that everything that happened after the big bang could be explained by natural sciences, including the development of the human mind and human consciousness. 
It is interesting that most naturalists among philosophers are not aware that the commitment to naturalism automatically makes them physicalists, materialists, and reductionists as well. When the assumption of naturalism is that the universe was created in the big bang, physics is the science to describe this event. Indeed physics knows a lot of details about the big bang. Physical models of the big bang allow physicists to make numerous predictions about the current status of the universe. And indeed, many of these predictions can actually be observed, proving the general correctness of the model. But on the other hand physics is far from being complete. About 30 years ago, it was discovered that besides ordinary matter-that is the substance humans and everything that we experience is made of - there are two more forms of matter, namely dark matter and dark energy. ${ }^{1}$ Even worse, it was discovered that ordinary matter makes up only $5 \%$ of all matter in the universe; 95\% is dark matter and dark energy. But physics even has open questions about ordinary matter. There are two great theories, which apply to ordinary matter, namely quantum theory and the general theory of relativity. Both theories yield excellent results. But these two theories are mutually contradictive so at least one theory must be wrong, maybe both. Quantum theory is used to describe the constituting elements of ordinary matter, namely atoms and elementary particles. The formalism achieves this with great precision but it remains unclear what the formalism means. In conclusion, one can say that physicists know only something about the smallest part of the universe, namely ordinary matter, but physicists don't really know what this ordinary matter is. Anyway, the lack of knowledge about the real character of matter does not prevent us from gaining a lot of knowledge about the principles that are relevant for our normal environment, the world of our sensual experience. That having been said, it has to be emphasized that indeed physics is the basis of all natural sciences and therefore physics is the fundament of naturalism. Consequently naturalism incorporates physicalism and every naturalist must automatically be a physicalist as well.

As indicated before, physics claims that the universe was created in the big bang. Physics also claims that in the big bang only one kind of substance was created, namely matter. As we just have shown, so far three different kinds of matter are known: dark matter, dark energy, and ordinary matter. It is absolutely possible that still more kinds of matter might be discovered in the future. Nevertheless, the claim of physics is that only one substance was created in the big bang and that all kinds of this substance obey the laws of physics. Even though we don't know what dark matter and dark energy are, we know that they have effects on the universe and on ordinary matter. That is the reason why they were discovered. But these effects are negligible in the world of our direct experience. Dark matter only has an effect on the scale of galaxies and dark energy only has an effect on the scale of the entire universe. On the other hand, this means that neither dark matter nor dark energy can have any effect on anything on earth. The fact that there are no effects in the world of our experience is precisely the reason why dark matter and dark energy were not discovered earlier. That is also the reason that neither dark matter nor dark energy can play any role in the explanation of the human mind or human consciousness. That is also the reason that no additional kind of matter could ever play any role for the explanation of anything on earth including humans. Consequently, physics and the other natural sciences, which are all based on physics, claim to explain every possible phenomenon in the world of our direct experience in terms of ordinary matter and interactions between material constituents. So far this explanation works very well. Consequently, physicalism includes materialism and every naturalist who automatically is a physicalist must automatically be a materialist as well.

Now the next step is obvious. Naturalism, physicalism and materialism claim that any object is made of matter. That must be the case because matter was the only substance created in the big bang. Actually, because 
of the negligible effect of dark matter and dark energy on anything on earth, we can even say that every object of our direct experience is made of ordinary matter. But if every object of our direct experience is made of ordinary matter that automatically means that it can be reduced to matter. The laws of nature don't allow any gap. There cannot "emerge" any objects or any phenomena that cannot be reduced to their material constituents. This would lead automatically to substance dualism. But substance dualism is ontologically impossible within naturalism because naturalism claims that only one substance was created in the big bang, namely matter. The emergence of any different substance would require new laws of nature and/or the abandoning of the current laws of nature. But that would be in contradiction to our definition of naturalism, namely that naturalism can explain everything based on the current laws of nature. But might there still be an alternative? If substance dualism is impossible within naturalism, there might exist the so-called "property dualism.” Property dualism claims that the human mind and human consciousness/phenomenal experiences are properties which are related to the brain but cannot be reduced to the brain. First, we have to ask what a property is. A property is always related to an object. Only objects can have properties. ${ }^{2}$ A property describes any kind of effect that the object has. Let us look at some examples. The object sun has the properties of temperature and brightness because the sun heats and illuminates the solar system. The earth has the property of mass because the earth attracts all objects on earth with gravitational force. Most objects have the property of color because they reflect a specific part of white light with which they are hit. We see that a property is always connected to an object and is an expression for the effects the object has. Consequently, per definition a property has no genuine ontological status but is always linked to an object and consequently can always be reduced to an object. That makes property dualism logically impossible. Even if the human mind and human consciousness/phenomenal experiences were just properties of the brain, they would be automatically materialistic and reducible to the brain. At any rate, the human mind and human consciousness are not just properties of the brain; they are special configurations or special abilities of the brain. Consequently, materialism includes reductionism and every naturalist who automatically is a physicalist and a materialist must automatically be a reductionist as well.

Based on naturalism, the author has explained the human mind and human consciousness materialistically (Prost 2016; 2017). The human mind is our language-representing neural network and human consciousness is the capability of humans to include verbal thinking in conscious processing. That proves that physicalism, materialism, and reductionism are correct. But that also means that the human mind and human consciousness are elements of the physical world and obey the laws of physics. As said before, it is disputed if these laws are completely deterministic or if some indeterminacies can occur. Consequently, we must now examine what free will is and whether free will is possible in any case.

\section{Free Will}

Since we are dealing with a composite term here, it is necessary to analyze both parts of it. Starting with the term "will," we may follow John Locke who describes the will as a power of mind which "urges us to start or desist, continue, or conclude diverse activities of the mind and movements of our body and indeed merely by a thought or any preference on the part of the mind which orders or effectively commands the compliance or noncompliance of this or that operation” (Locke 1998). In this definition, Locke accurately described various aspects of the human volition. Initially the will is a mental phenomenon as it happens inside the mind. Furthermore, the will provokes something: This can either be another mental phenomenon, or thought, or it may be a physical phenomenon, thus involving the movement of at least one body part. Moreover, Locke states 
that a deliberate omission of a mental or corporeal operation is also controlled by will. By virtue of the will, humans are thus capable of thinking or stopping further deliberation and they are also able to initiate or omit bodily actions. From this description, it follows that volition is a form of decision. Linguistically and analytically decision requires alternative possibilities; only if alternatives are given is a decision possible and only if a decision is to be made is an act of will required. Therefore, volition can be defined as a mental act that decides for or against the execution of a mental or physical action and that initiates or omits this action. Such an act of volition requires the existence of possible alternatives as a sine qua non. ${ }^{3}$

So when can our will be defined as autonomous or free? Locke states:

So far as a man has power to think or not to think, to move or not to move, according to the preference or direction of his own mind, so far is a man free. Wherever any performance or forbearance are not equally in a man's power; wherever doing or not doing will not equally follow upon the preference of his mind directing it, there he is not free, though perhaps the action may be voluntary. (Locke 1998)

Thus, it is in man's power to choose an alternative: He can decide otherwise. Therefore, the notion of free will is not only tied to the principle of alternative possibilities, but it is also tied to the possibility of being able to do things "otherwise." The very notion of free will is therefore tied to the possibility of "to do otherwise" on a linguistic analytical level. This leads to another requirement for free will, namely that free will decisions have to be controlled. Only if a decision is under a person's full control can the decision be considered to be free.

Subsequently, many think that freedom of action is a condition of the free will. Freedom of action is generally seen as a situation in which one is not prevented from executing an intended action. The threat of an armed robber to a jeweler is a well-known example for the illustration of the dependence of freedom of volition on freedom of action: Under threat of armed force, the robber demands the handover of the jewelry. If the jeweler actually hands over the jewels, he does so willingly but certainly not voluntarily. Since the jeweler has the desire to survive, he reasonably has no other choice than to hand over the goods. This means that the jeweler has no freedom of action and thus no freedom of volition.

However, freedom of action is not always the precondition for freedom of volition. In the above example, both freedoms are intertwined but whereas freedom of action can by definition only relate to actions, Locke explicitly includes acts of thoughts in his definition of freedom of volition. The freedom of thoughts therefore requires the possibility of alternatives and also the possibility to consider these alternatives, but it does not require freedom of action in the sense of a direct possibility to act upon these thoughts.

It has been known to humans for more than 3000 years that freedom of volition requires a mental act but not necessarily a physical action. The Ten Commandments God gave to Moses make a clear distinction between thoughts and deeds. ${ }^{4}$ Not only do the Commandments prohibit the wicked deed itself (as in the 6th Commandment "thou shalt not commit adultery" and the 7th Commandment "thou shalt not steal"), but the nefarious thought is also forbidden (as the 9th Commandment "thou shall not covet your neighbor's wife" and the 10th Commandment "thou shalt not covet thy neighbors goods" illustrate). Nonetheless, the free will is commonly tied to accomplished actions especially regarding moral responsibility. This has two reasons: Firstly, unlike God, no human is able to read another person's mind, therefore, practical reasons forbid the prosecution of evil thoughts. ${ }^{5}$ The second reason lies mainly in the power of suspension as Locke has defined it. Due to this power, there is a chance to reconsider one's deliberate decisions before implementation and to reach another decision. ${ }^{6}$ 
In summary, we can note that the will is a mental phenomenon that can initiate or omit bodily actions or mental actions. This requires from a linguistic analytical point of view the existence of alternative possibilities, and freedom of will means that an alternative can actually be selected. The decision must be controlled. Therefore, the principle of being able to do things "somehow or otherwise" is also linguistically, analytically tied to the notion of freedom in the expression "free will." A human being has a free will if she or he may choose (mentally) between different alternatives. In conclusion, we can say that "free" means that the person has the option to choose and to control a decision. "Free" excludes that the decision was already fixed before it came up.

\section{The Physical Realization of Will Decisions and Why They Can Never Be Free}

Now we have to explain shortly how decisions are physically realized in the brain. As said before, the human mind is the language-representing neural network and human consciousness is the capability of humans to include verbal thinking in conscious processing. Consequently, human thinking is a conscious process of the brain in which special neural configurations, called neural patterns, are connected in order to perform neural processes. These are completely physical processes and obey the laws of physics. Let us now start with the assumption that these processes are completely determined. That is the view of the compatibilists. The compatibilists argue that a decision-making process can be considered to be free when it is based on good reasons. Good reasons again are based on convictions and beliefs, in other words on the character of the person. Now we have to emphasize that all mental entities including reasons, convictions, and beliefs are neurally realized in the brain as neural patterns. The entire character of a person is part of her neural network. Also the way in which a person thinks is realized as neural connections. So what happens when a decision has to be made? There is input in the form of the creation of new neural patterns. These new neural patterns now initiate a thinking process which is a neural process. This neural process uses old neural patterns (which actually represent convictions and beliefs) to create new neural patterns which now represent reasons. In the next step, these reasons initiate actions by sending signals along neural connections or are responsible for omitting actions. As we have seen, the entire decision-making process is realized as a physical neural process in the brain. When now the assumption is that all physical processes are completely deterministic, then also this neurally realized decision-making process must be completely deterministic.

The error of compatibilists is their faulty understanding of the different levels of language which the author has extensively outlined (Prost 2016). One level of language is the language of neuroscience. Neuroscience describes structures and activities of the brain on a cellular level. The cells of the brain which are responsible for all brain activity are called neurons. They are highly connected and are active by signal transmissions along these connections. In this language, a person can be described by her neural network. The neural network represents the person's capabilities including her way of thinking, the person's knowledge and memory, and also the person's convictions and beliefs, in short: the person's character. For a neuroscientist, these are all only neural connections. These neural connections are now responsible for the way how a person thinks and acts. Alternatively, the person's behavior can be described in ordinary language. Ordinary language would say that the person acts because her convictions gave her good reasons for her actions. The behavior of the person can also be described in psychological language. Psychological language would say that she acts because her education and her experience formed her conviction which she used in decision-making and which finally led to her decisions and actions. And the behavior can also be described in philosophical language. 
Philosophical language would say that the zeitgeist created an atmosphere that led to the development of the person's character and the convictions that she used to develop reasons which caused her decisions and actions. As we see everything is identical, the only differences are the different levels of language. Convictions or beliefs are neural patterns. The processing of these neural patterns leads to reasons which again are neural patterns. The education and the experiences of the person that led to the convictions and beliefs are also neurally realized and finally formed the neural patterns representing these convictions and beliefs. And if the neural patterns representing convictions and beliefs of many people are summarized, they form the zeitgeist which again is of course realized as neural patterns in many brains. ${ }^{7}$

The explanation proves that the argument of compatibilists that a will decision can be considered as free when it is based on good reasons is wrong and does not make the decision a free will decision. The character of a person is completely determined by her neural network. This neural network is completely determined by the genes, the education, and the experiences of the person. This again completely determines which convictions and beliefs are formed as neural patterns inside the brain of this person. This again completely determines which reasons the person will develop if different decision-making situations occur. And finally all this completely determines every move the person ever makes in her life. ${ }^{8}$ At no point in time, the person has ever the opportunity to choose from different alternatives. What the compatibilists do is to manipulate the term "free will" in an unacceptable way. As we have seen, the will is not free. But the will is based on reasons. Consequently, it can be denoted as "reasonable will." But reasonable is not the same as free. The reason why we believe that we have a free will and that we think that we can choose from different alternatives is that we are not aware what happens in our brain. We are so to speak just observers of the actions of our brain. Then, using ordinary language, we report about our experiences which are just observations of neural processes. In ordinary language, we can report that we have special convictions and beliefs. In ordinary language, we can report that our convictions and beliefs led us to certain reasons for our actions. Our missing knowledge about the activities on the neural level then makes us think that we have made free-will decisions. What we also see here is that the question whether conscious or unconscious parts of the brain play the decisive role in decision making is completely irrelevant. Both parts work completely deterministically. So even if indeed only the conscious part were responsible for our decisions, the decisions themselves would still be deterministic. As a consequence, we can now say that the compatibilists are wrong: Free will is not compatible with determinism.

Now we have to investigate whether the possible indeterminacies of quantum theory provide a justification for libertarianism and its claim of free will. The physical assumption would have to be that not all neural processes are deterministic. The physical assumption would have to be that quantum effects play a role in some of the neural processes and lead to indeterminacies. There are two caveats. Right now it is not clear whether quantum theory indeed is indeterministic. Currently there are four major interpretations of quantum theory (Copenhagen interpretation, GRW, ${ }^{9}$ Bohmian Mechanics, and Many-Worlds Interpretation (MWI)). Two of them (Copenhagen, GRW) are indeterministic; the two others are deterministic. At the time being, the most popular one is the deterministic MWI. Intuitively many physicists believe that no interpretation is correct and that ultimately a deterministic explanation will be found. The second caveat is that possible quantum indeterminacies only occur for single or few quantum objects. But even a single neuron contains millions of atoms and multiple neurons are involved in neural processing. It is very questionable whether quantum effects still play a role in these huge compounds. But let's assume they would. Then another effect comes into play: All indeterministic events are automatically random. Now we have to remember that our foregoing definition of 
free will requires that all free-will decisions must be controlled. Only if the decision maker controls a decision can the decision be considered to be free. But indeterminism and control are mutually exclusive. No event that is indeterministic can ever be controlled.

Because libertarians deny the claim of the mutual exclusion of the terms "indeterminism" and "control," we will prove it by applying the terms to physical systems. The critical terms are "indeterminism," "random/chance," and "control." Let us start with indeterminism and let us ask what kinds of systems in physics are indeterministic. One example of an indeterministic quantum system is a single excited atom. Excitation means here that one of the electrons is moved out of the ground state to higher level. After some time which seems to be absolutely random, the electron moves back to the ground state and a photon is emitted. Every single photon emission is indetermined and consequently it is uncontrolled. For all physical systems, "indeterminism" always means "random" and no indeterministic physical event can ever be "controlled." For "control," there must have been an antecedent event, which would have caused the emission. We can illustrate this principle with a different example. This example is the LASER (Light Amplification by Stimulated Emission of Radiation). In an LASER, there exist many atoms, which are first excited by a special mechanism. That means in an LASER one first creates a state in which many atoms are transferred to a state of excitation. If nothing else were done, the atoms would decay one after the other, and every decay would be indetermined and absolutely random, just like in our previous example. But now a mechanism is used, called stimulation, which involves introducing an external photon with the same frequency as the photons which are normally created in the decay. This external photon now stimulates an excited atom to decay and as it does so, to emit a photon. A chain reaction is initiated, and all the other excited atoms fall back to the ground state and emit their photons as well. In this example, every emission of a photon is now determined by the stimulating photon and the following chain reaction. The emission of photons is no longer random, but controlled by an external determined action, namely the stimulation. The examination of these two physical systems, single excited atom, and LASER, illustrates the denotation of the different terms. Every physical event, which is indetermined, like the photon emission by a single excited atom, is absolutely random. Every indetermined physical event happens by chance. Due to the nature of indeterminism, no indetermined event can ever be controlled. Our examples also show that every event, which is controlled, like the LASER, must be determined. Now the law of non-contradictory tells us that then every event, which is not determined, can never be controlled. Because will decisions are also physical processes, all these considerations apply to will decisions as well.

Now we have proved again that the terms "indeterminism" and "random” are analytically connected. This means that every event which is indeterministic must automatically be random. For the question about free will, it means that every indeterministic will decision happens by chance. We have proved that the terms "indeterminism" and "control" are mutually exclusive. Thus, one of the most critical requirements of libertarianism, namely the control of the will decision, is not fulfilled. In summary, one can now say that libertarianism which claims that all will decisions are indeterministic must accept that these decisions can never be controlled and that all possibly indeterministic will decisions will always happen by chance. As a conclusion, we see that also libertarianism cannot provide a free will.

Consequently, neither deterministic nor indeterministic mental actions, here decisions, can ever be controlled in a natural world. Consequently, free will is logically not possible in naturalism. 


\section{An Alternative for the Existence of a Free Will}

Now it has to be emphasized again that all considerations here are based on the assumption of naturalism which included substance monism. Indeed we can be quite sure that this assumption is correct to explain the human mind and human consciousness. But we have mentioned above that there might be two forms of naturalism, pure naturalism, and religious naturalism. As we also have mentioned, the vast majority of mankind believes in the existence of another substance, namely a non-material soul. If we regard the position of the Abrahamitic religions, we have to envision that the soul might be responsible for the human conscience and for moral decisions. Indeed we have to admit that based on our current knowledge about the physical, material world we cannot exclude the possibility that the big bang, which is regarded as the creation of the universe, was caused by a non-material entity called God, imposing rules that led to the further development of the universe including humans. And it is absolutely possible that at some point, namely after human developed the capability to speak and to think, God gave a soul to humans. If the soul existed in a separate non-material world and interacted with neural processes only in the case of moral decisions, we would not be able to measure such interactions. Also, we would not be able to detect a simultaneous violation of laws of physics, such as the conservation of energy. In conclusion, we have to admit that we can indeed reduce the human mind and human consciousness to physics and matter, but we cannot exclude the possibility that a non-material soul does exist. As a consequence, the solution of the mind-body problem and the reduction of the human mind to matter does not provide a solution for the question whether humans do have a free will. We only know that free will is logically not possible within pure naturalism, but there might be a religious naturalism which would allow a free will. But religious naturalism requires substance dualism and is no longer genuine naturalism.

\section{Notes}

1. Einstein has shown the equivalence of matter and energy. That is the reason dark energy can be considered a form of matter.

2. Now the question about possible properties of "ideas" could be posed. As the author has shown (Prost 2016) "ideas" are also material objects, they are language-representing neural patterns. Consequently, properties of "ideas" are also connected to material objects.

3. It should be added that the alternatives must be (supposedly) realistic. If somebody states her will to fly with superluminal velocity, which is physically impossible, this can only be perceived as a wish or desire, not as an act of volition. A different situation is given when someone wants to open a door and then finds out that this door is locked. In this case, she has anticipated the alternatives of "opening the door" versus "keeping the door closed" to be existent and has voluntarily decided for one alternative. That these alternatives in fact did not exist is irrelevant, as the alternatives existed mentally,

4. The following considerations are valid, regardless whether or not somebody believes in the biblical tradition.

5. Though prosecution seems likely if the technical means existed! If one were able to read minds and thereby establish that a sexual offender was planning to commit a crime, one would intervene in order to prevent the offender from executing his acts of thought.

6. Even God would be unlikely to judge one's desire as a sin if it was suppressed by this person's power of suspension.

7. It should be mentioned here that humans developed methods to realize language outside of their brains, namely in the form of writing. But it can be emphasized that no written word has any meaning if it is not realized as a neural pattern within at least one single human brain.

8. Of course, in dependence of her environment and her experiences.

9. Named after the physicists G. Ghirardi, A. Rimini, and T. Weber.

\section{Works Cited}

Clarke, Randolph. Libertarian Accounts of Free Will. New York: Oxford University Press, 2005. 
---. "Libertarian Views: Critical Survey of Noncausal and Event-Causal Accounts of Free Agency.” Ed. Kane, Robert. The Oxford Handbook of Free Will. New York: Oxford University Press, 2002.

Dennett, Daniel C. Elbow Room. Oxford: Oxford University Press, 2002.

---. Freedom Evolves. London: Penguin Books, 2004.

Frankfurt, Harry G. The Importance of What We Care about. New York: Cambridge University Press, 1988.

Ginet, Carl. On Action. New York: Cambridge University Press, 1990.

---. "Reasons Explanation of Action: Causalist versus Noncausalist Accounts.” Ed. Kane, Robert. The Oxford Handbook of Free Will. New York: Oxford University Press, 2002.

Kane, Robert. The Oxford Handbook of Free Will. New York: Oxford University Press, 2002.

---. Free Will. New York: Oxford University Press, 2005.

Kant, Immanuel. Groundwork of the Metaphysics of Morals. Radford: Wilder Publications, 2008.

Libet, Benjamin. Mind Time. Harvard: Harvard University Press, 2005.

Locke, John. An Essay Concerning Human Understanding. London: Penguin Classics, 1998.

Mele, Alfred. Free Will and Luck. New York: Oxford University Press, 2006.

O’ Connor, Timothy. "Libertarian Views: Dualist and Agent Causal Theories.” Ed. Kane, Robert. The Oxford Handbook of Free Will. New York: Oxford University Press, 2002/1.

---. Persons and Causes: The Metaphysics of Free Will. New York: Oxford University Press, 2002/2.

Prost, Michael. Die Entschlüsselung des menschlichen Bewusstseins (The Mystery of Human Consciousness Unraveled). Wiesbaden: Springer VS, 2016.

---. "The Solution to the Mind-Body Problem: The Complete Reduction of the Human Mind to Matter, Namely to the Brain.” Sociology and Anthropology, Vol. 5, No. 4, 2017.

Roth, Gerhard. The Long Evolution of Brains and Minds. New York: Springer, 2013.

Strawson, Galen. Freedom and Belief. Oxford: Oxford University Press, 2010.

---. “The Bounds of Freedom.” Ed. Kane, Robert. The Oxford Handbook of Free Will. New York: Oxford University Press, 2002. 